\title{
Differentiation between Clinically Noninflammatory Granulomatous Lobular Mastitis and Noncalcified Ductal Carcinoma in situ Using Dynamic Contrast-Enhanced Magnetic Resonance Imaging
}

\author{
Ning Qu Yahong Luo Tao Yu \\ Department of Radiology, Cancer Hospital of China Medical University, Liaoning Cancer Hospital and Institute, \\ Shenyang, China
}

\section{Keywords}

Mastitis · Ductal carcinoma in situ - Magnetic resonance imaging $\cdot$ Nonmass enhancement $\cdot$ Clustered ring enhancement

\begin{abstract}
Introduction: Challenges in differentiation between clinically noninflammatory granulomatous lobular mastitis (GLM) and noncalcified ductal carcinoma in situ (DCIS) remain. Objective: To identify the dynamic contrast-enhanced magnetic resonance imaging (DCE-MRI) characteristics contributing to their differential diagnosis. Methods: A total of 33 clinically noninflammatory GLM and 36 noncalcified DCIS were retrospectively analyzed in the study. Internal enhancement of a nonmass enhancement (NME) lesion was divided into clustered enhanced ring (absence/presence), and clustered enhanced ring (presence) was further classified as small and large ring based on the optimal cutoff value. The 5th Breast Imaging and Data System MRI descriptors were used for assessing the other DCE-MRI characteristics. Multivariate analysis including variables with significant differences in univariate analyses was conducted to identify the independent predictors. The discriminative abilities of different predictors and their combination were compared by area under the receiver-operating characteristic curves (AUCs). Results: An NME lesion was seen more commonly in clinically noninflammatory GLM than in noncalcified DCIS ( $p=0.003)$. DCE$M R I$ characteristics with significant differences in univariate analyses included NME size, clustered enhanced ring (ab-
\end{abstract}

sence/presence), ring size, initial increase and kinetic characteristics for the differentiation between these two entities presenting as NME lesion. Clustered enhanced ring (presence) was further classified as small ( $\leq 7 \mathrm{~mm}$ ) or large ring (>7 mm). Multivariate analysis revealed that internal enhancement and initial increase were identified as significant independent predictors, and the AUC of their combination achieved the highest value of 0.867 ( $95 \% \mathrm{Cl}, 0.748-0.943)$. Conclusions: An NME lesion with a large ring is more highly suggestive of clinically noninflammatory GLM.

() 2020 S. Karger AG, Basel

\section{Introduction}

Granulomatous lobular mastitis (GLM) is a benign chronic inflammatory entity of the breast with unknown etiology and characterized by the presence of noncaseating granulomas with microabscess formation confined to the breast lobule [1]. GLM presents varied findings on mammography including irregular mass, asymmetry, normal finding and less architectural distortion, and no associated suspicious calcifications is a mammographic characteristic in almost all GLM [2-7]. Ductal carcinoma in situ (DCIS) is a noninvasive malignancy characterized by a proliferation of malignant epithelial duct cells confined to mammary ducts without demonstrable evidence of invasion through the basement membrane into the surrounding stroma [8]. Although the typical mammographic finding of DCIS is microcalcifications, a consid- 
erable proportion of DCIS are associated with noncalcified findings such as mass, asymmetry, even normal finding $[8,9]$. According to the presence or absence of calcifications on mammography, DCIS can be classified as calcified or noncalcified subtypes. Plainly, mammography can hardly discriminate GLM from noncalcified DCIS. Fortunately, a part of GLMs are easily differentiated from noncalcified DCIS because of their inflammatory features such as pain, tenderness, erythema, and sinus formation clinically [7]. However, for GLM without inflammatory features, it is difficult to distinguish it from noncalcified DCIS only depending on clinical manifestations and mammographic finding; moreover, ultrasound (US) is unhelpful in the differential diagnosis because the US findings of two conditions are heterogeneous and nonspecific $[10,11]$. To date, the definitive diagnosis of GLM can only be confirmed by histopathology [7]. Openexcision biopsy and fine needle aspiration are not recommended due to serious complications and low diagnostic rate, respectively $[3,10,12]$. Although the diagnostic rate is up to $96 \%$ for GLM using core needle biopsy with US [3], it is invasive after all.

Although DCIS of the breast is a nonobligate precursor to invasive breast cancer $[13,14]$, patients diagnosed as having DCIS should still first be treated with surgical excision because currently lesions with an indolent behavior cannot be discriminated from those with a higher risk of progression to invasive breast cancer $[15,16]$. For GLM, nonsurgical management is preferred, surgery is exclusively for the patient with refractory response to conservative therapy $[7,17]$. Therefore, it is essential to differentiate GLM from DCIS before treatment.

Dynamic contrast-enhanced magnetic resonance imaging (DCE-MRI) has become a well-established and valuable modality with the highest sensitivity for breast cancer detection. Furthermore, DCE-MRI demonstrated an excellent diagnostic performance to clarify equivocal noncalcified breast findings detected on conventional imaging (mammography, US) [18]. In light of this knowledge, we hypothesized that DCE-MRI may contribute to the differentiation between clinically noninflammatory GLM and noncalcified DCIS. Therefore, in the present study, we investigated whether DCE-MRI is an effective noninvasive modality for the differentiation between clinically noninflammatory GLM and noncalcified DCIS and identified the DCE-MRI characteristics contributing to the differential diagnosis.

\section{Materials and Methods}

\section{Patients}

This retrospective study was approved by the institutional review board of the Cancer Hospital of the China Medical University, and informed consent was waived because of the retrospective
Table 1. Comparison of the lesion types between clinically noninflammatory GLM and noncalcified DCIS on MRI

\begin{tabular}{llll}
\hline Lesion types & $\begin{array}{l}\text { Clinically noninflam- } \\
\text { matory GLM }\end{array}$ & $\begin{array}{l}\text { Noncalcified } \\
\text { DCIS }\end{array}$ & $p$ \\
\hline NME & 31 & 23 & 0.003 \\
Mass & 2 & 13 & \\
\hline
\end{tabular}

nature. Initially, 50 GLM lesions proved by histopathology after surgery in 49 consecutive patients (bilateral GLM was evaluated as 2 lesions in 1 patient) between January 2014 and March 2019 were included. Of these 49 patients, 5 lesions in 5 patients were excluded due to no breast DCE-MRI before the operation in our institution. Another 12 lesions in 11 patients were excluded due to the patients suffering from clinically inflammatory manifestations including pain, tenderness, erythema, sinus formation and skin ulcer. Finally, 33 GLM lesions in 33 women were enrolled in this study. We randomly selected 100 DCIS lesions diagnosed by postoperative histopathology during the same period. The exclusion criteria were as follows:(a) no available mammography or DCEMRI in our institution; (b) the presence of calcifications in the DCIS lesions on mammography. Thus, there were 36 noncalcified DCIS lesions in 36 women included in this study. The mean ages in women with clinically noninflammatory GLM and noncalcified DCIS were $34.0 \pm 4.9$ years (range, $26-43$ years) and $49.1 \pm 10.2$ years (range, $34-71$ years), respectively, showing a statistically significant difference $(p<0.001)$.

\section{Breast MRI Protocol}

All breast MRI examinations were performed with a 1.5-T system (Sigma Excite HD; GE Healthcare, Chicago, IL, USA) with an 8-channel high-definition breast coil with the patient placed in the prone position. Firstly, sagittal fat-suppressed T2-weighted images of the bilateral breasts were acquired, the following parameters were used: 4,040-ms repetition time (TR), 81-ms echo time (TE), echo train length of 19,5-mm slice thickness, 1-mm slice gap, 220 $\times 220$-mm field of view (FOV), $320 \times 224$ matrix, number of excitations of 2, 18 images and imaging time of $1 \mathrm{~min} 41 \mathrm{~s}$ for each breast. Then we used an axial three-dimensional fat-suppressed gradient-echo T1-weighted sequence (VIBRANT; GE Medical Systems) for the precontrast and dynamic postcontrast imaging with 42-s (per phase) temporal resolution, with the following parameters: $6.2-\mathrm{ms} \mathrm{TR}, 3.0-\mathrm{ms} \mathrm{TE}$, flip angle of $10^{\circ}, 3.2-\mathrm{mm}$ slice thickness, no gap, $360 \times 360-\mathrm{mm}$ FOV, $256 \times 350$ matrix, $48 \mathrm{im}$ ages each phase, a total acquisition time of $5 \mathrm{~min} 36 \mathrm{~s}$. Gadolinium chelate contrast material was administered by intravenous bolus at a dose of $15 \mathrm{~mL}$ and at a rate of $2.5 \mathrm{~mL} / \mathrm{s}$, followed by a $20-\mathrm{mL}$ saline flush using an automatic power injector.

\section{MRI Interpretation}

The two radiologists specializing in breast imaging who were blinded to the histopathological diagnoses evaluated the lesions on a processing workstation (ADW 4.3; GE Healthcare) in consensus. According to Breast Imaging and Data System (BI-RADS) 5th edition, the lesions were divided into three categories: focus, mass and nonmass enhancement (NME). None of the lesions in this study presented with focus and the great majority of the lesions showed NME. Hence, the lesions in this study were classified as two categories: NME and mass. The evaluating items of NME were as follows: size (the largest diameter), distribution, internal enhancement on 
Table 2. Results of the univariate analysis of the DCE-MRI characteristics of clinically noninflammatory GLM and noncalcified DCIS presenting as NME

\begin{tabular}{llcc}
\hline MRI characteristics & $\begin{array}{l}\text { Clinically noninflammatory } \\
\text { GLM }\end{array}$ & Noncalcified DCIS & $p$ \\
\hline NME size, mm & $52.1 \pm 12.9(28-80)$ & $38.6 \pm 13.9(13-67)$ & 0.001 \\
Distribution & 10 & 13 & 0.075 \\
$\quad$ Segmental/linear & 21 & 10 & 0.001 \\
$\quad$ Nonsegmental/linear & 28 & 11 & 0.002 \\
Clustered enhanced ring & 3 & 12 & 0.049 \\
$\quad$ Absence & 7 & 15 & \\
$\quad$ Presence & 24 & 8 & \\
$\quad$ Snitial increase & 2 & 16 & \\
$\quad$ Fast & 29 & & \\
Kinetic characteristics & & & \\
$\quad$ Persistent & & & \\
$\quad$ Plateau/washout & & & \\
\hline
\end{tabular}

the second phase of the DCE sequence; initial increase and kinetic characteristics based on time signal intensity curve. The distribution was classified as linear or segmental pattern consistent with mammary duct and nonlinear or segmental pattern. The internal enhancement was categorized as absence or presence of a clustered enhanced ring (regardless of the size of rings), and ring size was documented as the long diameter of the largest ring in the lesion. The initial increase (slow/intermediate, fast) and kinetic characteristics (persistent, plateau/washout) were based on BI-RADS 5th edition [19]. For a mass-like lesion, the evaluation was based on the descriptors of the 5th BI-RADS MRI lexicon. The region of interest was obtained manually on the area with the greatest enhancement of the second phase of the DCE sequence, and region of interest size ranged from 3 to 5 pixels.

\section{Statistical Analysis}

The Kolmogorov-Smirnov test was used for analyzing normality of continuous variables such as age, size of NME lesion and ring size. Continuous variables with normal distribution were expressed as means \pm standard deviation, and the comparisons of these variables between two groups were conducted with the independent sample $t$ test. Dichotomous variables including lesion type and characteristics of NME lesion (distribution, internal enhancement, initial increase and kinetic characteristics) were analyzed by the $x^{2}$ test. The optimal cutoff value of ring size for the differential diagnosis was determined based on the maximal Youden index by receiver-operating characteristic (ROC) curve, and the discriminative ability was assessed by the area under the ROC curve (AUC). Large and small rings were defined on the basis of the optimal cutoff value of the ring size. Variables with statistically significant differences in univariate analysis were included in multivariate regression analysis to identify the independent predictors for an NME lesion. Internal enhancement of NME was incorporated into a multivariate logistic regression equation in the form of no ring, small or large ring. Multivariate logistic regression analysis adopted a backward stepwise regression method. The discriminative abilities of independent predictors alone and their combination were compared by AUCs. $p<0.05$ was considered as statistically significant difference by a two-tailed test. Analyses were performed using SPSS version 19.0 and MedCalc version 15.0
Table 3. Results of the multivariate analysis of the DCE-MRI characteristics of clinically noninflammatory GLM and noncalcified DCIS presenting as NME

\begin{tabular}{lclr}
\hline Predictors & Odds ratio & $\begin{array}{l}\text { 95\% confidence } \\
\text { interval }\end{array}$ & $p$ \\
\hline Ring size & & & \\
$\quad$ Large & 1 & & \\
$\quad$ Small & 9.995 & $1.817-54.976$ & 0.008 \\
$\quad$ None & 29.739 & $4.593-192.555$ & $<0.001$ \\
Initial increase & & & \\
$\quad$ Fast & 1 & & \\
$\quad$ Slow/intermediate & 5.017 & $1.107-22.730$ & 0.036 \\
\hline
\end{tabular}

\section{Results}

Histological classification of the nuclear grade for 36 noncalcified DCIS: 13 were low-grade, 15 were intermediate-grade, and 8 were high-grade.

A comparison of the lesion types between clinically noninflammatory GLM and noncalcified DCIS is shown in Table 1. An NME was detected more frequently in clinically noninflammatory GLM than in noncalcified DCIS $(p=0.003)$. The results of the univariate analysis of the DCE-MRI characteristics of clinically noninflammatory GLM and noncalcified DCIS presenting as NME are presented in Table 2. The NME size of clinically noninflammatory GLM was significantly greater than that of noncalcified DCIS $(52.1 \pm 12.9$ vs. $38.6 \pm 13.9 \mathrm{~mm}, p=0.001)$. A clustered enhanced ring was present more commonly in clinically noninflammatory GLM than in noncalcified DCIS. Clinically noninflammatory GLM more often had a fast initial increase and plateau/washout kinetic charac- 


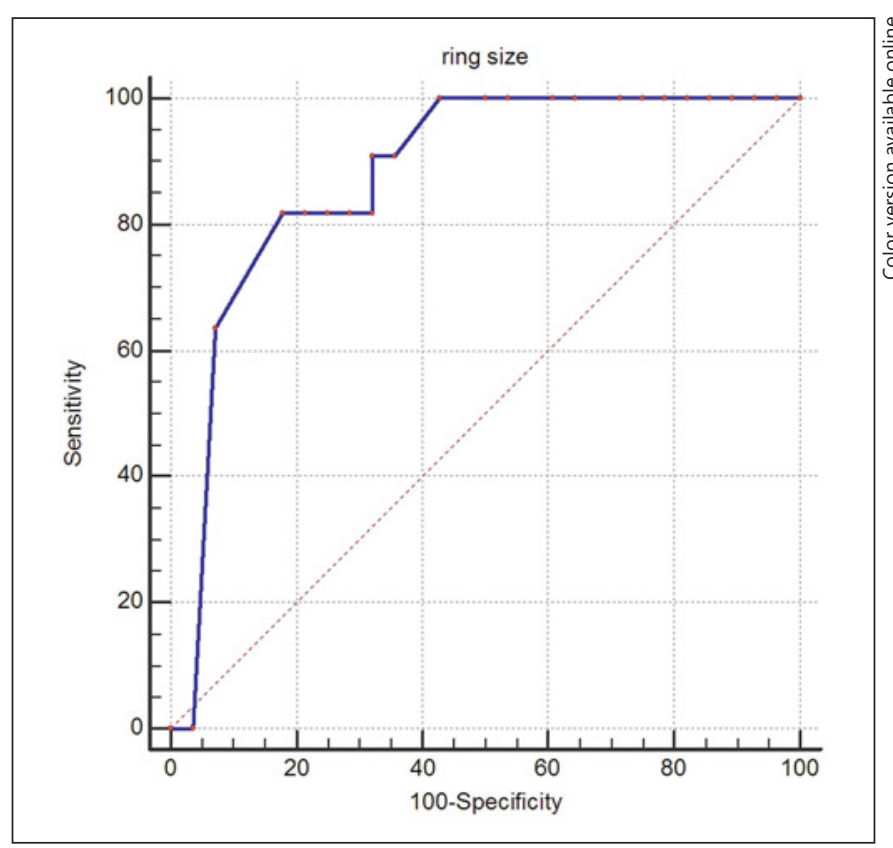

Fig. 1. A graph showing the ROC curve for ring size in the differentiation between clinically noninflammatory GLM and noncalcified DCIS.

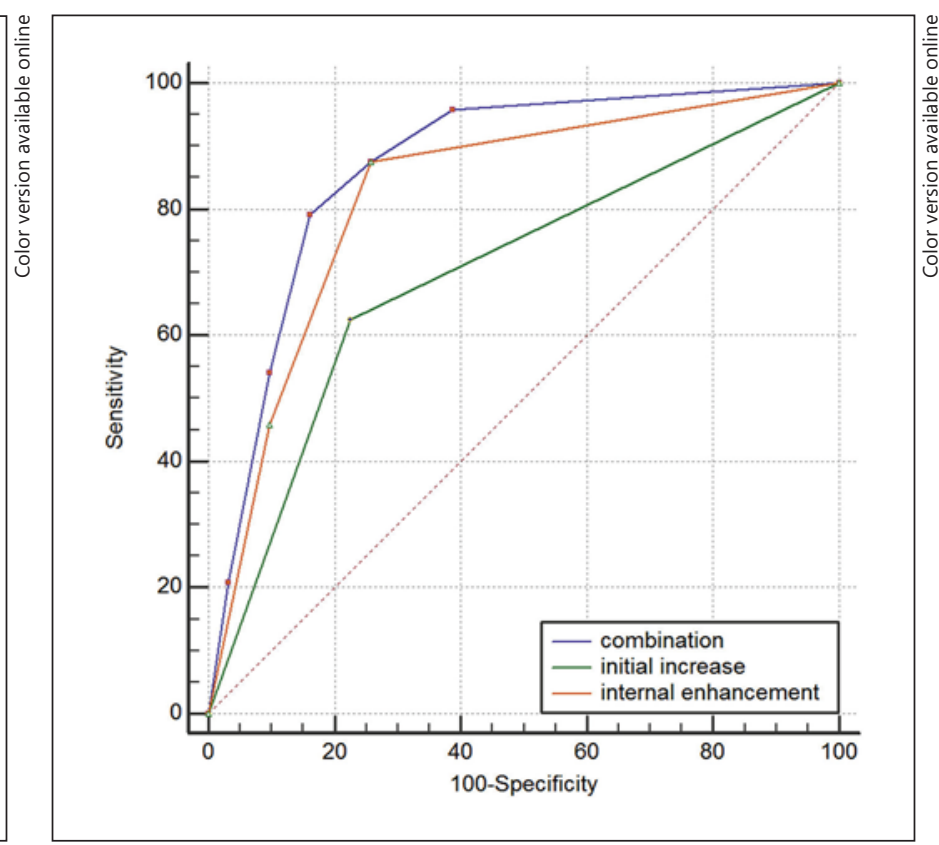

Fig. 2. The ROC curves of using internal enhancement, initial increase and their combination for distinguishing between clinically noninflammatory GLM and noncalcified DCIS presenting as NME.
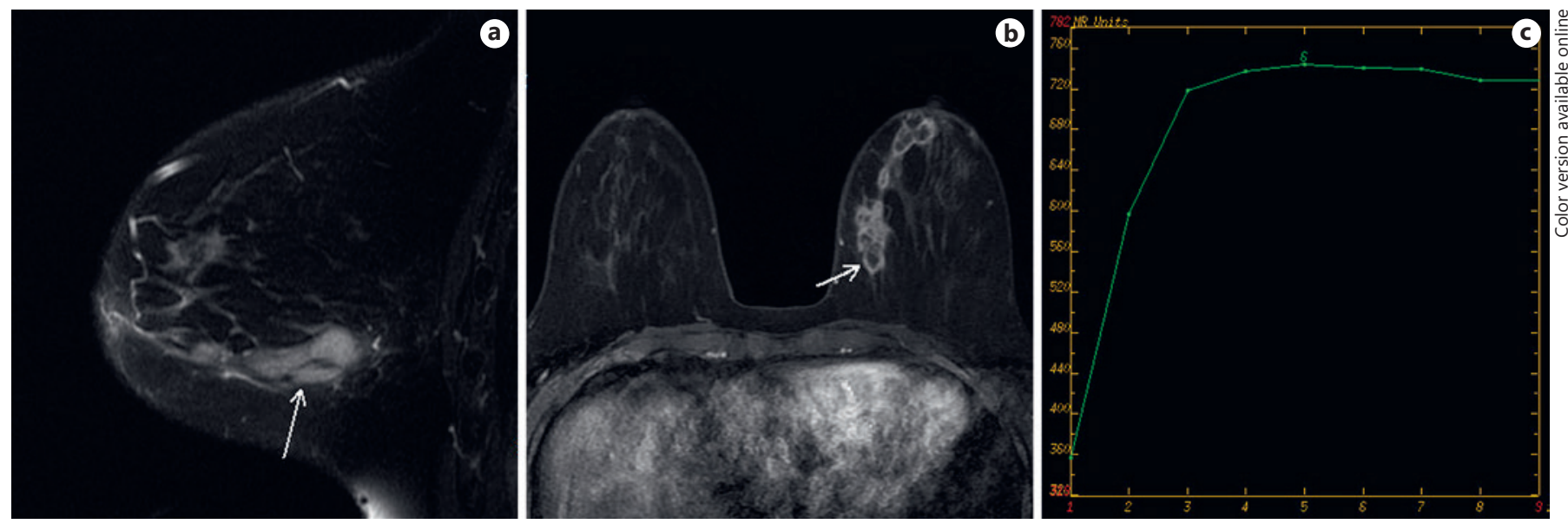

Fig. 3. A 35-year-old woman with granulomatous lobular mastitis in her left breast. a Sagittal T2-weighted fat-suppressed image of the left breast shows a lesion presenting with segmental distribution and high-signal intensity (arrow). b Axial postcontrast T1- weighted image in the second phase shows large rings $(>7 \mathrm{~mm})$ with heterogeneous sizes clustered together (arrow). c Time-signal intensity curve shows fast initial increase with plateau pattern in the delayed phase. teristics than noncalcified DCIS. Ring size in clinically noninflammatory GLM was significantly larger than that in noncalcified DCIS $(12.4 \pm 5.1$ vs. $6.8 \pm 1.4 \mathrm{~mm}, p<$ 0.001 ). The optimal cutoff value of ring size was determined as $7 \mathrm{~mm}$ with the AUC of 0.878 (95\% confidence interval [CI], 0.734-0.961) (Fig. 1), yielding sensitivity and specificity of 81.8 and $82.1 \%$, respectively, for the diagnosis of clinically noninflammatory GLM. The results of the multivariate analysis of DCE-MRI characteristics of clinically noninflammatory GLM and noncalcified DCIS presenting as NME are shown in Table 3. Internal enhancement and initial increase were identified as significant independent predictors. The AUCs of internal enhancement, initial increase and their combination for differentiating clinically noninflammatory GLM from noncalcified DCIS were 0.825 (95\% CI, 0.699-0.914), 0.700 (95\% CI, 0.561-0.816) and 0.867 (95\% CI, 0.7480.943) (Fig. 2). The AUC of their combination was sig- 

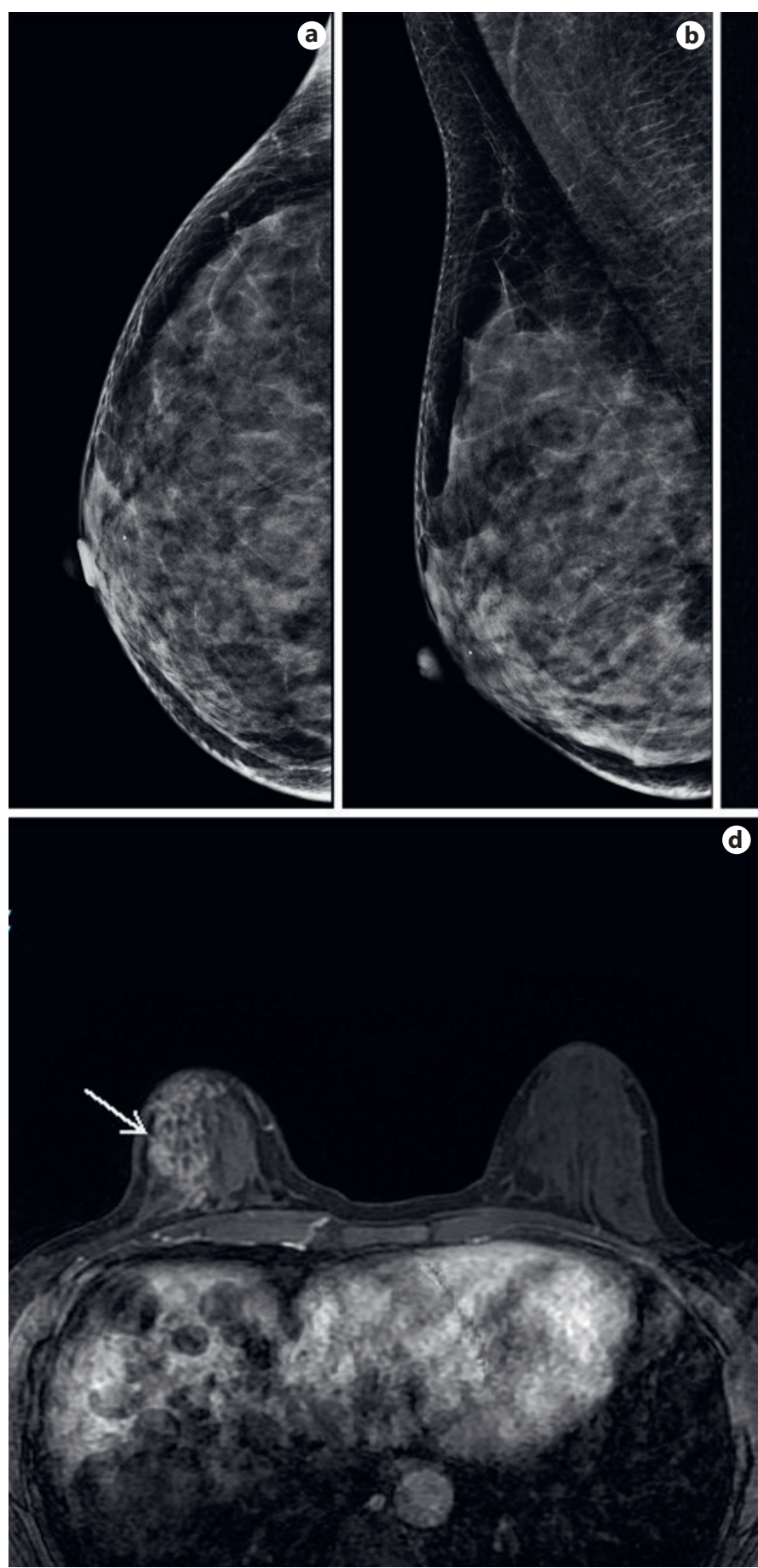

Fig. 4. A 38-year-old woman with extensive ductal carcinoma in situ (intermediate-grade nuclear) in her right breast. Right breast craniocaudal (a) and mediolateral (b) mammogram views show normal findings. c Sagittal T2-weighted fat-suppressed image of the left breast shows a lesion presenting with segmental distribution and slightly high-signal intensity (long arrow), with ductal

nificantly larger than that of the initial increase ( $p=$ $0.0013)$ and comparable to that of internal enhancement $(p=0.1352)$. The AUCs between internal enhancement and initial increase did not differ significantly $(p=0.3147)$.

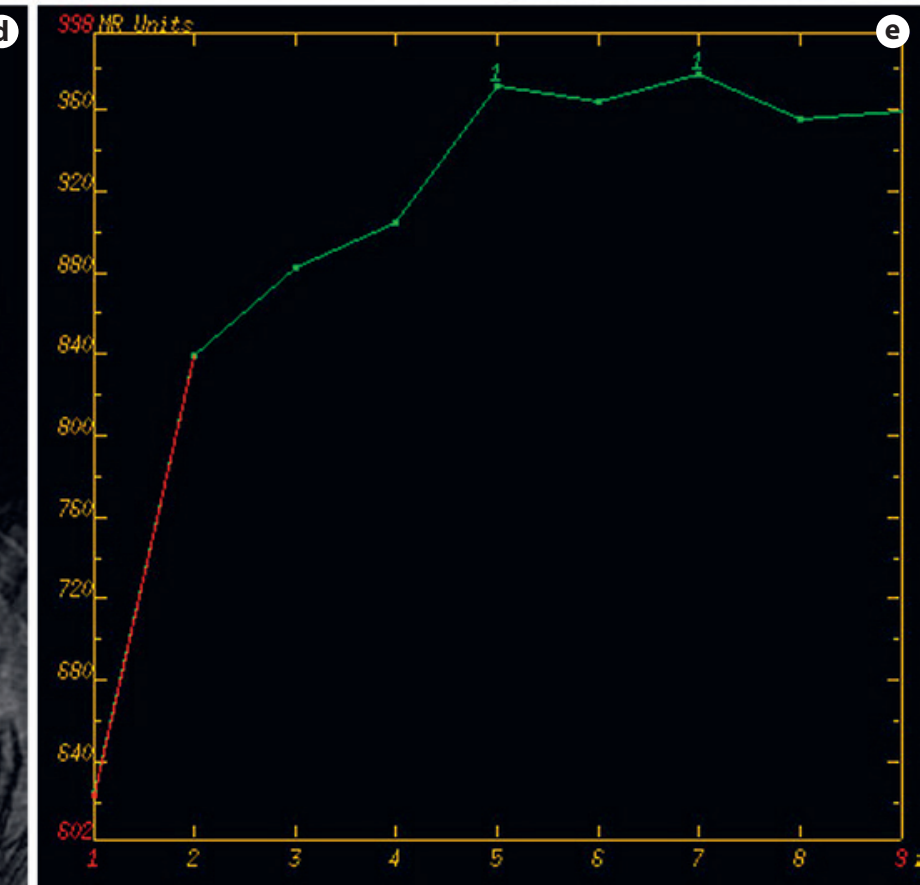

ectasia showing high-signal intensity of liquid in front of the lesion (short arrow). d Axial postcontrast T1-weighted image in the second phase shows small rings $(\leq 7 \mathrm{~mm})$ with homogeneous sizes clustered together (arrow). e Time-signal intensity curve shows slow initial increase with persistent pattern in the delayed phase.

Representative MR images and corresponding mammographic images of clinically noninflammatory GLM and noncalcified DCIS are shown in Figures 3 and 4, respectively. 


\section{Discussion}

Our study demonstrated that the vast majority of clinically noninflammatory GLM presented with an NME lesion $(31 / 33,93.9 \%)$. The results of multivariate logistic regression analysis revealed that internal enhancement and initial increase were identified as two independent predictors for the differentiation between clinically noninflammatory GLM and noncalcified DCIS showing an NME lesion. Moreover, the discriminative performance of combination internal enhancement with initial increase achieved the highest AUC of 0.867 in comparison with that of internal enhancement or initial increase alone. Our results indicate that DCEMRI can contribute substantially to the differentiation between clinically noninflammatory GLM and noncalcified DCIS.

Although GLM lesions demonstrated a wide spectrum of MRI findings, they typically appeared as NME $[2,19-$ 22]. The proportion of clinically noninflammatory GLM showing NME in this study was comparable to that in the study by Aslan et al. [21] (31/33, 93.9\% vs. 33/36, 92.3\%). The analysis of lesion types showed that clinically noninflammatory GLM were significantly correlated to a higher fraction of NME lesion, while noncalcified DCIS showed a higher proportion of mass lesion. However, the majority of lesion types in noncalcified DCIS were NME that was consistent with those reported in the literature [23-25]. The published reports demonstrated that there were no significant differences in terms of morphology and enhancement characteristics on breast MRI between calcified and noncalcified DCIS [26, 27]. Kim et al. [28] also reported that morphological appearance and initial uptake or kinetic patterns of delayed phase were not associated with four different mammographic patterns.

Clustered ring enhancement was first proposed by Tozaki et al. [29] in 2006 and referred to as a finding in which minute ring enhancements were clustered within the heterogeneously enhanced lesion. It was included in the BIRADS 5th edition as a new term describing internal enhancement of an NME lesion. It is believed to be the enhanced effect of the periductal stroma and ductal wall [29]. According to the recent study, clustered ring enhancement was found to be the second strongest malignant predictor with odds ratios of 3.601 and 4.71 [30, 31]. Nevertheless, for the lesion with clustered ring enhancement, in addition to the risk of DCIS and invasive breast cancer, benign lesions such as complex sclerosis, papilloma and fibrocystic disease should also be taken into account [32]. The positive predictive value of clustered ring enhancement for malignancy varied greatly with the range of $41.9-100 \%$ due to the different patient populations between studies [30, 32-34]. The clustered enhanced ring used in the current study was referred to as multiple rings clustered together regardless of ring size, including the aforementioned clustered ring enhancement and larger rings clustered together which was not included in the 5th BI-RADS MRI lexicon. ROC curve analysis revealed that the optimal cutoff value for discriminating clinically noninflammatory GLM from noncalcified DCIS was $7 \mathrm{~mm}$ with high discriminative performance, sensitivity and specificity. Clinically noninflammatory GLM presenting as an NME more often showed a large ring greater than the cutoff value of $7 \mathrm{~mm}$ than noncalcified DCIS $(23 / 28$, $82.1 \%$ vs. $2 / 11,18.2 \%)$. According to the results of multivariate analysis, the probability of noncalcified DCIS was 9.995 times that of clinically noninflammatory GLM for NME lesions with small rings and the risk of noncalcified DCIS was 29.739 times that of clinically noninflammatory GLM for NME lesions without any rings. Pairwise comparison of ROC curves showed that the discriminative ability of internal enhancement alone was close to that of the combination of two significant predictors. The difference between the ring sizes of these two conditions may be due to their different pathological mechanisms: small rings of DCIS are the enhancement of periductal stroma and ductal wall [29], while large rings of GLM correspond to aseptic confluent abscess [35].

Regardless of the lesion type, the most common initial uptake in DCIS is fast, which is seen in $49-68 \%$ of cases [13]. However, Baltzer et al. [36] reported that the malignancy presenting as an NME primarily displayed slow/ intermediate initial uptake mainly resulting from a lower level of tumor neoangiogenesis; thus, malignant NME lesions could not be distinguished from benign NME lesions on the basis of initial uptake. In this study, among noncalcified DCIS presenting as an NME, $65.2 \%$ of cases showed slow/intermediate initial uptake which was in line with the result in the aforementioned study [36]. The proportion of nonhigh nuclear grade of noncalcified DCIS was as high as $77.8 \%[28,36]$ which was in agreement with the result that noncalcified DCIS tended to have a nonhigh nuclear grade $[27,37]$. The contrast enhancement in DCIS is attributed to the presence of tumor angiogenesis in the stroma surrounding involved ducts by DCIS [38]. The previous studies showed that highgrade DCIS had significantly higher tumor angiogenesis than non-high-grade DCIS $[16,39]$. In addition, the degree of initial increase is associated with the amount of tumor angiogenesis. It has been shown that the higher the nuclear grade in DCIS, the higher the probability of fast initial increase [38]. Therefore, a high proportion of noncalcified DCIS with nonhigh nuclear grade might be a dominant reason for a low fraction of fast initial increase in this study. Yilmaz et al. [20] reported that only $4.8 \%$ of GLM presented with a fast initial increase, whereas all lesions enhanced fast in the early phase in another study [2]. In this study, $77.4 \%$ of clinically noninflammatory 
GLM showed a fast initial increase. The remarkable discrepancy between studies may be explained as a result of the different stages of inflammation. Hence, though initial increase was determined as one of the independent predictors by multivariate analysis, it should be used with caution for the differential diagnosis of clinically noninflammatory GLM and noncalcified DCIS until it is validated by a larger sample in the future. Fortunately, pairwise comparison of ROC curves revealed that the addition of initial increase to internal enhancement did not significantly improve the discriminative ability. In other words, the influence of initial increase on the predictive result is not significant.

Clinically noninflammatory GLM had a significantly larger NME size than noncalcified DCIS owing to the intrinsic characteristics of inflammation itself which tends to spread along mammary ducts [39]. It is inappropriate to apply to the result that a lesion size of $1 \mathrm{~cm}$ or larger can act as the only independent indicator of malignancy for NME lesions [32] for the differential diagnosis of these two conditions in our study. In some cases of DCIS, larger NME sizes result from the extensive growth pattern of the lesion and the overestimation of the actual extent on DCE-MRI due to additional areas of enhancement related to the great number of proliferative changes. Therefore, NME size should be interpreted with caution and used in combination with the other features. Segmental/ linear distribution was seen more commonly in noncalcified DCIS although there was no statistical difference regarding the distribution between the two groups in the study. Segmental distribution is defined as "a triangular area of enhancement, apex pointing to the nipple, suggesting a duct or its branches" [23]. Multivariate analyses showed that segmental distribution had the highest odds ratios of 4.739 and 5.1 for discriminating benign and malignant NME lesions [30,31]. The positive predictive value for malignancy of segmental distribution ranged from 67 to $100 \%$ [34]. In the 5th edition lexicon, ductal distribution has been changed to the term "linear." Linear distribution is divided into two patterns: single line or branching. The positive predictive values differed significantly between branching pattern and single line ( $74.7 \mathrm{vs.}$ $8 \%$ ) [40]. A branching line with the size of $1 \mathrm{~cm}$ or more was significantly more frequently associated with malignancy than a single line smaller than $1 \mathrm{~cm}$ [31]. Linear distribution with single line pattern smaller than $1 \mathrm{~cm}$ was recommended to be assigned to the BI-RADS 3 category $[31,40]$. Consistent with results from a previous study [23], 30.4\% of non-calcified DCIS presenting as NME lesion showed the persistent enhancement. Although univariate analysis showed that kinetic characteristics had significantly statistical difference between the two groups in this study, which was hardly of value in clinical practice because the kinetic characteristics of GLM varied with different pathological stages.

We acknowledge the limitations in the present study. Firstly, this is a retrospective study. And our sample size was relatively small due to the rarity of GLM, especially GLM with no inflammatory features detected clinically. Nevertheless, the proportion of females with GLM who had no clinical findings suggestive of inflammation was relatively high because our hospital predominantly focuses on cancers. Further data collection from multicenter trials are needed to address the differential diagnosis between clinically noninflammatory GLM and noncalcified DCIS showing a mass-type lesion. Secondly, a diffusionweighted imaging (DWI) sequence was not included in this study. Although there was no statistically significant difference in apparent diffusion coefficient (ADC) values between GLM and invasive breast cancer [20,21, 41], the ADC value in a DCIS lesion was significantly above that of invasive ductal carcinoma [13], so the ADC value of DCIS might be higher than that of GLM. Moreover, a central hyperintensity on DWI images with a lower ADC value of the central region is a valuable feature for the diagnosis of inflammatory breast lesion with abscess which is different from invasive breast cancer with central necrosis showing a peripheral hyperintensity on DWI images with a lower ADC value of the wall [42]. Therefore, we will do further study to investigate the potential value of DWI and the ADC value for the differentiation between clinically noninflammatory GLM and noncalcified DCIS.

\section{Conclusions}

In conclusion, the vast majority of clinically noninflammatory GLM exhibit NME lesions on DCE-MRI, and the characteristic of internal enhancement classified as no, small or large ring is determined as the most significant independent predictor for differentiating it from noncalcified DCIS. A large ring is more highly suggestive of clinically noninflammatory GLM, while no or a small ring is more highly predictive of noncalcified DCIS. Therefore, DCE-MRI can act as an effective noninvasive method to differentiate clinically noninflammatory GLM from noncalcified DCIS, avoiding unnecessary surgical excision to some extent for patients with clinically noninflammatory GLM.

\section{Statement of Ethics}

This study was approved by the institutional review board of the Cancer Hospital of the China Medical University, and informed consent was waived because of the retrospective nature. 


\section{Disclosure Statement}

The authors have no potential conflicts of interest to disclose.

\section{Funding Sources}

This study received funding from Liaoning Natural Science Foundation (2019-KF-01-02) and National Nonprofit Industry Research of China (201402020). This was used to pay consulting fees for language and statistics.

\section{Author Contributions}

Y.L. conceived the project, and N.Q. designed the study. Y.L. and N.Q. analyzed the imaging. N.Q. collected the patients' characteristic data, prepared figures and wrote this manuscript. T.Y. analyzed data and prepared tables. Y.L. as the corresponding author approved the final version to be submitted.

\section{References}

1 Benson JR, Dumitru D. Idiopathic granulomatous mastitis: presentation, investigation and management. Future Oncol. 2016 Jun; 12(11):1381-94.

2 Gautier N, Lalonde L, Tran-Thanh D, El Khoury M, David J, Labelle M, et al. Chronic granulomatous mastitis: Imaging, pathology and management. Eur J Radiol. 2013 Apr; 82(4):e165-75.

3 Aghajanzadeh M, Hassanzadeh R, Alizadeh Sefat S, Alavi A, Hemmati H, Esmaeili Delshad MS, et al. Granulomatous mastitis: Presentations, diagnosis, treatment and outcome in 206 patients from the north of Iran. Breast. 2015 Aug;24(4):456-60.

4 Memis A, Bilgen I, Ustun EE, Ozdemir N, Erhan Y, Kapkac M. Granulomatous mastitis: imaging findings with histopathologic correlation. Clin Radiol. 2002 Nov;57(11):1001-6.

5 Dursun M, Yilmaz S, Yahyayev A, Salmaslioglu A, Yavuz E, Igci A, et al. Multimodality imaging features of idiopathic granulomatous mastitis: outcome of 12 years of experience. Radiol Med (Torino). 2012 Jun;117(4):52938.

6 Fazzio RT, Shah SS, Sandhu NP, Glazebrook KN. Idiopathic granulomatous mastitis: imaging update and review. Insights Imaging. 2016 Aug;7(4):531-9.

7 Barreto DS, Sedgwick EL, Nagi CS, Benveniste AP. Granulomatous mastitis: etiology, imaging, pathology, treatment, and clinical findings. Breast Cancer Res Treat. 2018 Oct; 171(3):527-34

8 Stomper PC, Connolly JL, Meyer JE, Harris JR. Clinically occult ductal carcinoma in situ detected with mammography: analysis of 100 cases with radiologic-pathologic correlation. Radiology. 1989 Jul;172(1):235-41.

9 Ikeda DM, Andersson I. Ductal carcinoma in situ: atypical mammographic appearances. Radiology. 1989 Sep;172(3):661-6.

10 Pluguez-Turull CW, Nanyes JE, Quintero CJ, Alizai H, Mais DD, Kist KA, et al. Idiopathic granulomatous mastitis: manifestations at multimodality imaging and pitfalls. Radiographics. 2018 Mar-Apr;38(2):330-56.

11 Wang LC, Sullivan M, Du H, Feldman MI, Mendelson EB. US appearance of ductal carcinoma in situ. Radiographics. 2013 Jan-Feb; 33(1):213-28

12 Hovanessian Larsen LJ, Peyvandi B, Klipfel N Grant E, Iyengar G. Granulomatous lobular mastitis: imaging, diagnosis, and treatment.
AJR Am J Roentgenol. 2009 Aug;193(2):57481

13 Greenwood HI, Heller SL, Kim S, Sigmund EE, Shaylor SD, Moy L. Ductal carcinoma in situ of the breasts: review of MR imaging features. Radiographics. 2013 Oct;33(6):156988.

14 Yeong J, Thike AA, Tan PH, Iqbal J. Identifying progression predictors of breast ductal carcinoma in situ. J Clin Pathol. 2017 Feb; 70(2):102-8.

15 Margenthaler JA, Vaughan A. No Surgery for low-grade ductal carcinoma in situ? JAMA Surg. 2015 Aug;150(8):746.

16 Kuhl CK, Schrading S, Bieling HB, Wardelmann E, Leutner CC, Koenig R, et al. MRI for diagnosis of pure ductal carcinoma in situ: a prospective observational study. Lancet. 2007 Aug;370(9586):485-92.

17 Al-Khaffaf B, Knox F, Bundred NJ; AI-Khaffaf B. Idiopathic granulomatous mastitis: a 25-year experience. J Am Coll Surg. 2008 Feb; 206(2):269-73.

18 Bennani-Baiti B, Bennani-Baiti N, Baltzer PA. Diagnostic performance of breast magnetic resonance imaging in non-calcified equivocal breast findings: results from a systematic review and meta-ananlysis. PLoS One. 2016 Aug;11(8):e0160346.

19 Chu AN, Seiler SJ, Hayes JC, Wooldridge R, Porembka JH. Magnetic resonance imaging characteristics of granulomatous mastitis. Clin Imaging. 2017 May-Jun;43:199-201.

20 Yilmaz R, Demir AA, Kaplan A, Sahin D, Ozkurt E, Dursun M, et al. Magnetic resonance imaging features of idiopathic granulomatous mastitis: is there any contribution of diffusion-weighted imaging in the differential diagnosis? Radiol Med (Torino). 2016 Nov; 121(11):857-66

21 Aslan H, Pourbagher A, Colakoglu T. Idiopathic granulomatous mastitis: magnetic resonance imaging findings with diffusion MRI. Acta Radiol. 2016 Jul;57(7):796-801.

22 Kocaoglu M, Somuncu I, Ors F, Bulakbasi N, Tayfun C, Ilkbahar S. Imaging findings in idiopathic granulomatous mastitis. A review with emphasis on magnetic resonance imaging. J Comput Assist Tomogr. 2004 Sep-Oct; 28(5):635-41.

23 Raza S, Vallejo M, Chikarmane SA, Birdwell RL. Pure ductal carcinoma in situ: a range of MRI features. AJR Am J Roentgenol. 2008 Sep;191(3):689-99.
24 Chadashvili T, Ghosh E, Fein-Zachary V, Mehta TS, Venkataraman S, Dialani V, et al. Nonmass enhancement on breast MRI: review of patterns with radiologic-pathologic correlation and discussion of management. AJR Am J Roentgenol. 2015 Jan;204(1):21927.

25 Jansen SA, Newstead GM, Abe H, Shimauchi A, Schmidt RA, Karczmar GS. Pure ductal carcinoma in situ: kinetic and morphologic MR characteristics compared with mammographic appearance and nuclear grade. Radiology. 2007 Dec;245(3):684-91

26 Scott-Moncrieff A, Sullivan ME, Mendelson EB, Wang L. MR imaging appearance of noncalcified and calcified DCIS. Breast J. 2018 May;24(3):343-9.

27 Mun HS, Shin HJ, Kim HH, Cha JH, Kim H. Screening-detected calcified and non-calcified ductal carcinoma in situ: differences in the imaging and histopathological features. Clin Radiol. 2013 Jan;68(1):e27-35.

28 Kim JA, Son EJ, Youk JH, Kim EK, Kim MJ, Kwak JY, et al. MRI findings of pure ductal carcinoma in situ: kinetic characteristics compared according to lesion type and histopathologic factors. AJR Am J Roentgenol. 2011 Jun;196(6):1450-6.

29 Tozaki M, Igarashi T, Fukuda K. Breast MRI using the VIBE sequence: clustered ring enhancement in the differential diagnosis of lesions showing non-masslike enhancement. AJR Am J Roentgenol. 2006 Aug;187(2):313-21.

30 Yang QX, Ji X, Feng LL, Zheng L, Zhou XQ, Wu Q, et al. Significant MRI indicators of malignancy for breast non-mass enhancement. J XRay Sci Technol. 2017;25(6):1033-44.

31 Asada T, Yamada T, Kanemaki Y, Fujiwara K, Okamoto S, Nakajima Y. Grading system to categorize breast MRI using BI-RADS 5th edition: a statistical study of non-mass enhancement descriptors in terms of probability of malignancy. Jpn J Radiol. 2018 Mar;36(3): 200-8.

32 Uematsu T, Kasami M. High-spatial-resolution 3-T breast MRI of nonmasslike enhancement lesions: an analysis of their features as significant predictors of malignancy. AJR Am J Roentgenol. 2012 May;198(5):1223-30.

33 Yuen S, Uematsu T, Masako K, Uchida Y, Nishimura T. Segmental enhancement on breast MR images: differential diagnosis and diagnostic strategy. Eur Radiol. 2008 Oct; 18(10):2067-75. 
34 Aydin H. The MRI characteristics of nonmass enhancement lesions of the breast: associations with malignancy. Br J Radiol. 2019 Apr;92(1096):20180464.

35 Oztekin PS, Durhan G, Nercis Kosar P, Erel S, Hucumenoglu S. Imaging findings in patients with granulomatous mastitis. Iran J Radiol. 2016 May;13(3):e33900.

36 Baltzer PA, Benndorf M, Dietzel M, Gajda M, Runnebaum IB, Kaiser WA. False-positive findings at contrast-enhanced breast MRI: a BI-RADS descriptor study. AJR Am J Roentgenol. 2010 Jun;194(6):1658-63.

37 Kim JH, Ko ES, Kim DY, Han H, Sohn JH, Choe DH. Noncalcified ductal carcinoma in situ: imaging and histologic findings in 36 tumors. J Ultrasound Med. 2009 Jul;28(7):90310.

38 Facius M, Renz DM, Neubauer H, Böttcher J, Gajda M, Camara O, et al. Characteristics of ductal carcinoma in situ in magnetic resonance imaging. Clin Imaging. 2007 Nov-Dec; 31(6):394-400

39 Tan H, Li R, Peng W, Liu H, Gu Y, Shen X. Radiological and clinical features of adult non-puerperal mastitis. Br J Radiol. 2013 Apr; 86(1024):20120657.

40 Machida Y, Tozaki M, Shimauchi A, Yoshida T. Two distinct types of linear distribution in nonmass enhancement at breast MR imaging: difference in positive predictive value between linear and branching patterns. Radiology. 2015 Sep;276(3):686-94.

41 Zhang L, Hu J, Guys N, Meng J, Chu J, Zhang $\mathrm{W}$, et al. Diffusion-weighted imaging in relation to morphology on dynamic contrast enhancement MRI: the diagnostic value of characterizing non-puerperal mastitis. Eur Radiol. 2018 Mar;28(3):992-9.

42 Wang L, Wang D, Fei X, Ruan M, Chai W, Xu $\mathrm{L}$, et al. A rim-enhanced mass with central cystic changes on MR imaging: how to distinguish breast cancer from inflammatory breast diseases? PLoS One. 2014 Mar; 9(3):e90355. 